\title{
ANA MARÍA LORANDI Y EL PRIMER CONGRESO INTERNACIONAL DE ETNOHISTORIA
}

\author{
ANA MARIA LORANDI AND THE FIRST INTERNATIONAL CONGRESS \\ OF ETNOHISTORY
}

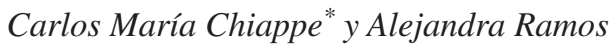

\begin{abstract}
Ana María Lorandi fue una investigadora pionera en su país pero el impacto de sus investigaciones y capacidad de gestión se extendió más allá de Argentina y alcanzó toda la región andina. Un recorrido por su trayectoria se ve atravesado por los vaivenes políticos de Argentina, las transformaciones disciplinares en las Ciencias Sociales y los desarrollos institucionales orientados a la formación de investigadores y a la difusión y financiación de sus trabajos. Mostraremos de qué manera puede leerse esta articulación y cómo recuperar reflexivamente estos elementos a partir de un hito en la carrera de Ana María, la organización del Primer Congreso Internacional de Etnohistoria, evento que contribuyó a la consolidación nacional y regional de la Etnohistoria. Para ello retomaremos la voz de Ana María en entrevistas realizadas en los últimos años y presentaremos material inédito de la organización del Congreso.
\end{abstract}

Palabras claves: Ana María Lorandi, Congreso Internacional de Etnohistoria, Andes.

Ana María Lorandi was a pioneer researcher in her country and the impact of her research and management capacity extended beyond Argentina and reached the Andean region. A journey through her trajectory is traversed by the political ups and downs of Argentina - in line with the region -, the disciplinary transformations in the Social Sciences and the institutional developments oriented to the training of researchers and the diffusion and financing of their works. We will show how this articulation can be read and how recover reflexively these elements from a milestone in the career of Ana María, the organization of the first International Congress of Ethnohistory, an event that contributed to the national and regional consolidation of Ethnohistory. For this we recover the voice of Ana María in interviews carried out in the last years and we present unpublished material of the organization of the Congress Key words: Ana María Lorandi, International Congress of Ethnohistory, Andes.

Nunca se termina de aprender a investigar. Nuevo tema, nuevo desafio, la historia se repite... Se repiten las dudas, la ansiedad, la desorientación, la excitación, el esfuerzo por expresar lo que queremos decir, siempre y cuando sepamos qué es lo que queremos decir. $Y$, por cierto, también el placer, el inmenso placer de investigar y aprender cada día algo nuevo. $Y$ sobre todo tener paciencia para emprender la ardua tarea de procesar los datos. La aventura de descubrir y la monotonía de clasificar u ordenar son inseparables, algo recurrente tanto si tienes veinte años cuando comienzas, o a los noventa (si llegas) cuando tal vez terminas.

Ana María Lorandi (2013:187)

\section{Introducción}

Nacida en Cañada de Gómez, provincia de Santa Fe, el recorrido académico de Lorandi se inició en 1960, cuando se recibió de Profesora de Historia por la Universidad del Litoral. Por aquella época, el contacto con Alberto Rex González la llevó a volcarse a la arqueología, participando de los trabajos de campo en el NOA. Su crecimiento profesional le permitió luego -entre 1965 y 1985 dirigir investigaciones arqueológicas en Santiago del Estero, por las que se la considera una de las principales promotoras del conocimiento históricoantropológico de la región (Taboada 2015).

Lorandi se doctoró muy joven, en 1967, en una época en que "era usual que fuera el corolario de una larga carrera académica". Esto la habría favorecido en varios aspectos de su profesión, como el poder ingresar como investigadora del Consejo Nacional de Investigaciones Científicas y Técnicas (CONICET) y el hacerse un lugar entre los andinólogos referentes del escenario académico internacional (Boixadós y Bunster 2016:10). Un año antes, durante la dictadura de Onganía, Lorandi había renunciado a su puesto docente en la Universidad del Litoral, pasando

\footnotetext{
* Universidad de Buenos Aires, Facultad de Filosofía y Letras, Instituto de Ciencias Antropológicas, Sección Etnohistoria. Argentina. Correo electrónico: carlosmariachiappe@ gmail.com
} 
luego a formar parte de la Universidad de La Plata (Torres, Gesteira y Hirsch 2011:155).

Por la misma época, conforme la arqueología argentina se volcaba a su faceta más dura, Lorandi empezó a interesarse por la etnohistoria. La transición de la Lorandi arqueóloga a la etnohistoriadora fue, sin embargo, lenta. Esta abarcó toda la década de 1970 y los años iniciales de la del 80, en un contexto político signado por la dictadura cívico-militar de 1976-1983. En este traspaso, el contacto con destacados investigadores del campo etnohistórico fue también decisivo. En primer lugar, está su vínculo con John Murra, al que conoció en el Congreso Internacional de Arte Rupestre en Huánuco (Perú, 1967), cuando este se hallaba difundiendo su modelo de verticalidad y complementariedad. En segundo lugar, su estancia en Francia en los 70, en donde se vinculó al equipo de Nathan Wachtel y realizó un posgrado en la École des Hautes Études (Ramos y Chiappe 2016).

El retorno de la democracia en 1983 trajo nuevos aires a las Ciencias Sociales argentinas. En la UBA se encaró una reforma de la docencia e investigación en antropología que incluyó al Instituto de Ciencias Antropológicas (ICA). En aquella oportunidad le fue ofrecido a Lorandi hacerse cargo del ICA. Su aceptación la alejó de la Universidad de La Plata, asumiendo la dirección desde 1984 hasta 1991. Lorandi llevó a cabo una profunda reforma en el ICA, que involucró la creación de nuevas secciones, entre ellas la de Etnohistoria y la Antropología Social. Estas operaban -y lo hacen todavía- como espacios de investigación especializada. A la par de su trabajo en este puesto de gestión, se preocupó por colaborar en la consolidación de la Etnohistoria a nivel regional. Las tareas llevadas a cabo por la Sección Etnohistoria (SE) se caracterizaron por presentar una perspectiva interdisciplinar que combina la Antropología, la Historia y la Arqueología y que fue cobrando mayores dimensiones a partir de la incorporación de nuevas áreas de estudio, así como de becarios y graduados. Desde entonces, la práctica de la etnohistoria en la UBA cobró gran dinamismo mediante diferentes materias y seminarios, proyectos, publicaciones y eventos académicos de alcance internacional (Zanolli et al. 2010, Zanolli, Ramos y Chiappe 2016).

Todos los que compartieron espacios y momentos con Ana María, desde sus primeros hasta sus últimos discípulos y colegas, coincidirían en señalarla como pionera en Argentina de una forma holística de investigar, que combinó los aportes de diferentes disciplinas, y formó una escuela caracterizada por el compromiso de trabajo, la honestidad intelectual, el compañerismo y la reciprocidad (Zanolli 2016, Boixadós y Bunster 2016). En nuestro caso, como investigadores interesados en el análisis de las prácticas en las que el conocimiento científico respecto de lo andino se produce y circula, además de refrendar las anteriores aseveraciones, reconocemos en Ana María una pionera de nuestro tema de estudio. En sus primeros trabajos tras embarcarse en la Etnohistoria se ocupó de las articulaciones entre Antropología e Historia y de la forma específica que adquirió en los Andes (cfr. Lorandi y Rodríguez Molas 1984, Lorandi y del Río 1992); este tópico permaneció como parte de sus intereses hasta sus escritos más recientes (cfr. Lorandi 2012).

En trabajos recientes hemos abordado la figura de Ana María Lorandi focalizando en su recorrido como docente e investigadora, en puestos de gestión y en su inserción en las redes académicas internacionales (Zanolli, Ramos y Chiappe 2016, Ramos y Chiappe 2016). A grandes rasgos, estos tópicos incluyen sus trabajos pioneros en arqueología del NO argentino (NOA); su labor etnohistórica, que ayudó a posicionar el Tucumán colonial como tema de investigación; la creación de la cátedra Sistemas Socioculturales de América II en la Universidad de Buenos Aires (UBA) y de otros espacios docentes de grado y posgrado; la formación de innumerables recursos humanos; la continua implementación de proyectos de investigación con diversas fuentes de financiamiento; su labor reformista al frente del Instituto de Ciencias Antropológicas de la UBA y la organización de eventos académicos de alcance internacional. En este pequeño escrito-homenaje quisiéramos focalizar en un hito fundamental para el desarrollo de los estudios andinos, el Primer Congreso Internacional de Etnohistoria (Buenos Aires, 1989).

\section{El Primer Congreso Internacional de Etnohistoria (I CIE)}

En 2015 y 2016, en el marco de dos proyectos individuales de investigación acerca de la evolución de los estudios andinos (Ramos 2016, Chiappe 2018), realizamos varias entrevistas a Ana María en su casa del barrio de San Cristóbal (C.A.B.A.). Entre otras cuestiones, nos interesaba conocer 
cómo se había organizado el I CIE en la Facultad de Filosofía y Letras de la UBA (Buenos Aires, 1989). Teníamos entonces la intención -y, ahora, la certeza de lograrlo- de publicar diversos materiales inéditos (cartas, circulares, resúmenes) originados alrededor de ese evento, los que veníamos leyendo a la par que organizándolos para su inclusión en el Archivo Institucional de la Sección Etnohistoria (AISE). La futura publicación precisaba de un exordio crítico y quién mejor que Ana María para darnos detalles y orientar el análisis. Los siguientes párrafos condensan información obtenida por medio de la pesquisa archivística y de aquellas charlas con ella.

En enero de 1983 se celebró en San Pedro de Atacama el Primer Simposio de Arqueología Atacameña. Lorandi presentó allí la ponencia "El borde oriental de la Puna de Tucumán prehispánica" en el simposio coordinado por John Murra y Jorge Hidalgo, titulado "Problemática etnohistórica de la Subárea Circumpuneña: Síntesis actualizada". En la versión impresa del programa y las conclusiones del simposio -publicada al año siguiente en Estudios Atacameños- quedó asentado el ofrecimiento de la autora para "gestionar la organización de un simposio de Etnohistoria Andina en Buenos Aires para un futuro próximo" (Universidad Católica del Norte 1984: 21). Es esta la primera mención publicada de lo que finalmente tomaría forma como el I CIE. Obsérvese que esto sucedió antes de que Lorandi se vinculase a la UBA, por lo que el interés en organizar este evento fue anterior a su asunción como directora del ICA.

Poco tiempo después de fundada la SE, su pequeño pero calificado equipo asumió el desafío de organizar el I CIE. Luego de una agitada gestación, que empezó en 1987 -recordemos que, en aquella época, toda comunicación se realizaba por correo postal-, el evento se organizó sobre la base de cinco simposios que trataban los temas centrales de la época: Rebeliones (Coord. Frank Salomon), Simbolismo y sincretismo religioso (Coord. Luis Millones), Estrategias de supervivencia (Coord. Thierry Saignes), Pasado y presente de las relaciones interétnicas en el Gran Chaco, Brasil central y Amazonia (Coord. Alejandra Siffredi); y Sociedad indígena y relaciones fronterizas en el área meridional de Argentina y Chile (siglos XVI-XIX) (Coord. Raúl Mandrini). Al que se sumaba un sexto simposio destinado a Comunicaciones (Cuarta Circular ICIE 1989, Libro de Resúmenes ICIE 1989).
El Comité Organizador ${ }^{1}$ dispuso, en el marco del Congreso, un homenaje a Gunnar Mendoza "en reconocimiento de su obra en el Archivo Nacional de Bolivia y por el constante, erudito y cordial apoyo que Ud. brinda a los investigadores que lo visitan" (Carta de Lorandi-del Río a Mendoza 1987). Se decidió homenajear también a John Murra "en mérito a su importante labor en torno de la Etnohistoria Andina y su influencia magistral sobre varias generaciones de especialistas que reconocen en Ud. al maestro y orientador singular" (carta de Lorandi-del Río a Murra 1987). La confirmación de Murra no se hizo esperar: "Acabo de recibir tu carta con la invitación al congreso INTERNACIONAL de etnohistoria... y qué programa... Claro que acepto y con emoción. Ahora tendré que planear alguna contribución que valga la pena" (carta de Murra a Lorandi 1988).

Entre los participantes se encontraron otras figuras extranjeras como Frank Salomon, Luis Millones, Thierry Saignes, Thomas Abercrombie, Silvia Arze, Eric Langer, Robin Wrigth, Amalia Castelli, Isabelle Combès, Gilles Riviere, Héctor Noejovich, Carlos Mamani, Carmen Bernand, Horacio Zapater, Jorge Hidalgo, Luis Galdames, Osvaldo Silva y Eduardo Téllez, entre otros (Libro de Resúmenes ICIE 1989). La comitiva chilena fue, como se ve, abundante. ${ }^{2}$

Como se destaca del contenido de las cartas guardadas en el AISE, el I CIE solo pudo concretarse por la férrea voluntad de la Comisión Organizadora y -en especial- de Ana María, ya que su realización coincidió con el estallido social y político que atravesaba el país. La hiperinflación, que se agudizaba mes a mes, impidió la financiación de todos los viajes y estadías programados, debiéndose cancelar las invitaciones para dictados de clases post-Congreso, ya que la Universidad no podía solventarlas. A pesar de estos inconvenientes, el evento tuvo una amplia convocatoria, recibiendo inesperadamente a más de 500 asistentes.

Aún hoy, y pese al evidente éxito del I CIE, resulta difícil entender cómo se impulsó su realización en una coyuntura tan volátil, que no permitía la más mínima previsión. ¿Por qué Ana María siguió con el plan original? La respuesta, más sencilla de lo imaginado, se relaciona con la voluntad del grupo:

Estábamos tan embaladas cuando ocurrió la renuncia [del presidente] Alfonsín, que no previmos lo que iba a pasar... La inflación crecía, pero la renuncia de Alfonsín es lo que la disparó. Era tal la inflación en ese 
Dr. JOHN MURRA

515 Dryden Road

ITHACA NoY. 14850 - U.S.A.

Querido John: rentes al Congreso.

Contesto a tu carta informándote de las últimas noticias refe-

1.- A Teresa Rojas ya le hablamos escrito unos días antes de tu carta porque tuvimos noticias de ella por Mandrini.

2.- El país estå en plena bancarrota. No sé si recuerdas a cuántos australes estaba el dólar en Septiembre/Octubre, creo que a A 8.- Pues ahora está a A 71.-1!!

Nos han suspendido la entrega de pasajes. La Comisión Fulbright nos comunica hoy que no acceden a nuestra solicitud y la Warner Green no nos respondió.

Con los fondos que recaudamos podremos pagarle el pasaje a Don Gunnar, pero desde New York casi nos parece imposible si las cosas no se alivian un poco.

En estos días están aquí Sanchez Albornoz y Herbert Klein, los consultamos y la única sugerencia fué que trates de hablar directamente con la Earner Green para ver si hay posibilidades. Nosotros nos comprometemos a pagar el alojamiento. Si nos quedan fondos o sucede un milagro, tal vez podamos contribuir con algo más.

Lamentamos muchísimo esta situación, pero te digo que este mes (un mes) la inflación fué del $40 \%$. Las cosas se nos escapan de las manos $y$ te pedimos mil disculpas pero no hallamos solución. Contamos, que a pesar de esto no dejarás de venir. El Congreso se está organizando bien, a pesar de estos problemas.

Un abrazo afectuoso.

\section{ANA MARIA}

Figura 1. Carta de Ana María Lorandi a John Murra, en donde se expresan las condiciones de realización del Congreso.

momento, que ni pagando en dólares -por anticipado- nos querían hacer la reserva en los hoteles donde se hospedarían los coordinadores y asistentes más reconocidos... Nosotras no habíamos organizado congresos, entonces, se nos desbordó de tal manera... ¡Pero le pusimos un ímpetu! ¡Fue terrible! Nunca nos imaginamos todo ese maremágnum de gente que vino... a esa Facultad que estaba casi vacía... fría... no había calefacción [...] en pleno julio... ¡solo pudimos ofrecer una copa de vino económica, de damajuana, porque no teníamos nada! Y en ese congreso -que fue el primero- aparecieron españoles, ;que no teníamos ni idea de cómo se habían enterado! De Bolivia y Perú, ¡lleno! (Lorandi 2015).

El I CIE fue un evento pionero que contribuyó a la consolidación nacional y regional de la Etnohistoria. Su valor como espacio de actualización y difusión de la disciplina ha quedado demostrado en su continuidad hasta el presente. ${ }^{3}$ Su relevancia estuvo dada por aspectos de diverso orden. En 
primer lugar, el de la coyuntura académica. Como dijimos, la SE es producto de una reorganización institucional tras la reapertura democrática. Sus líneas de investigación se anclan en tradiciones nacionales (como la impulsada por el reconocido arqueólogo A. Rex González) e internacionales (como parte del auge de las discusiones acerca de los vínculos entre Antropología e Historia). En segundo lugar, la coyuntura política. El I CIE se realizó en medio del proceso hiperinflacionario acontecido durante el gobierno de Raúl Alfonsín (1983-1989), lo que afectó notablemente su desarrollo. Si bien el Congreso no se suspendió, sí lo hicieron diversas actividades complementarias que hubieran posibilitado un mayor aprovechamiento de la concurrencia de investigadores de relieve internacional. En tercer lugar, la gestación de redes académicas. El I CIE representó un hito en la conformación de una red académica internacional en torno a la Etnohistoria andina, campo que revolucionó la investigación de la región y que continúa manteniendo una impronta interdisciplinar e internacional. En este sentido, el I CIE contribuyó a que el equipo de trabajo de la SE se instalara en el plano internacional.

\section{En Memoria}

Pese a los lógicos problemas de salud que cualquier persona de edad tiene, hasta el último año de vida Ana María seguía desarrollando diferentes actividades, siendo la más recordada el recibimiento del Doctorado Honoris Causa por la Universidad de Santiago del Estero, en mérito a su trayectoria $\mathrm{y}$, en especial, por sus aportes al desarrollo de la arqueología santiagueña. El paulatino y reciente retiro de los espacios de la UBA en los que se había desempeñado durante años, traspasados, ahora, a sus más conspicuos discípulos, no había morigerado un ápice su afán de conocer. Hace pocos años Ana María escribió, comentando una investigación en curso:

En esta etapa preliminar en la que me encuentro estoy profundizando el análisis de documentación producida por diversos personajes que participan de los acontecimientos de fines del siglo XVIII y comienzos del XIX. Algunos firmes defensores del absolutismo monárquico como Benito Mata Linares o el obispo San Alberto, otros que proponen una monarquía parlamentaria o moderada como Victoriano Villava, pero todos a su manera opuestos al sistema republicano, como sinónimos de anarquía política y de inversión de la jerarquía social. Sobre todo, en América, donde el temor a un hipotético gobierno de los indígenas no solo retarda la emancipación de la Corona de España, sino que refuerza la solidaridad interna de la sociedad no indígena o que no quiere identificarse con ella. No vale la pena insistir en la gigantesca tarea que tengo por delante: leer toneladas de libros y artículos sobre este período de transición y la República temprana tanto en América como en España, a lo que se suma la búsqueda de documentación que dé cuenta de los microprocesos locales sobre los que en este momento me gustaría indagar. Por tanto, en este momento me debato entre la incertidumbre acerca de la metodología más adecuada para abordar estos objetivos y la adrenalina que provoca un nuevo desafío (Lorandi 2013:198).

Efectivamente, cuando falleció Ana María, estaba en prensa un artículo concerniente a su trayectoria en donde destacábamos su mantenido vigor, volcado ahora a conocer la trayectoria de Juan Antonio Álvarez de Arenales (1770-1831) mediante su recorrido independentista por diversos espacios andinos que ella había abordado en sus investigaciones (Ramos y Chiappe 2016). Según nos comentó en la última de las entrevistas que le realizamos, el general Arenales era una figura que le permitía "hilvanar" a los actores de estos espacios desde una perspectiva biográfica inserta en la mirada más general que ella ayudó a desarrollar, la de la Antropología Histórica. Lo que implicaba atender simultáneamente a las lógicas nativas y a la profundidad temporal para abordar el estudio y la compresión de los procesos sociales regionales.

En 2014 Ana María participó del IX Congreso Internacional de Etnohistoria, realizado en la Universidad de Tarapacá, Arica. Allí estuvo tan vital como siempre, interactuando con sus pares, concurriendo a los simposios, visitando el Museo Arqueológico San Miguel de Azapa e interviniendo en la sesión de clausura. Conocida y reconocida por todos, recordamos cuánto disfrutó en aquella oportunidad, e intuimos que ella valoraría en gran medida este sentido homenaje en la revista Diálogo Andino de la misma Universidad. 


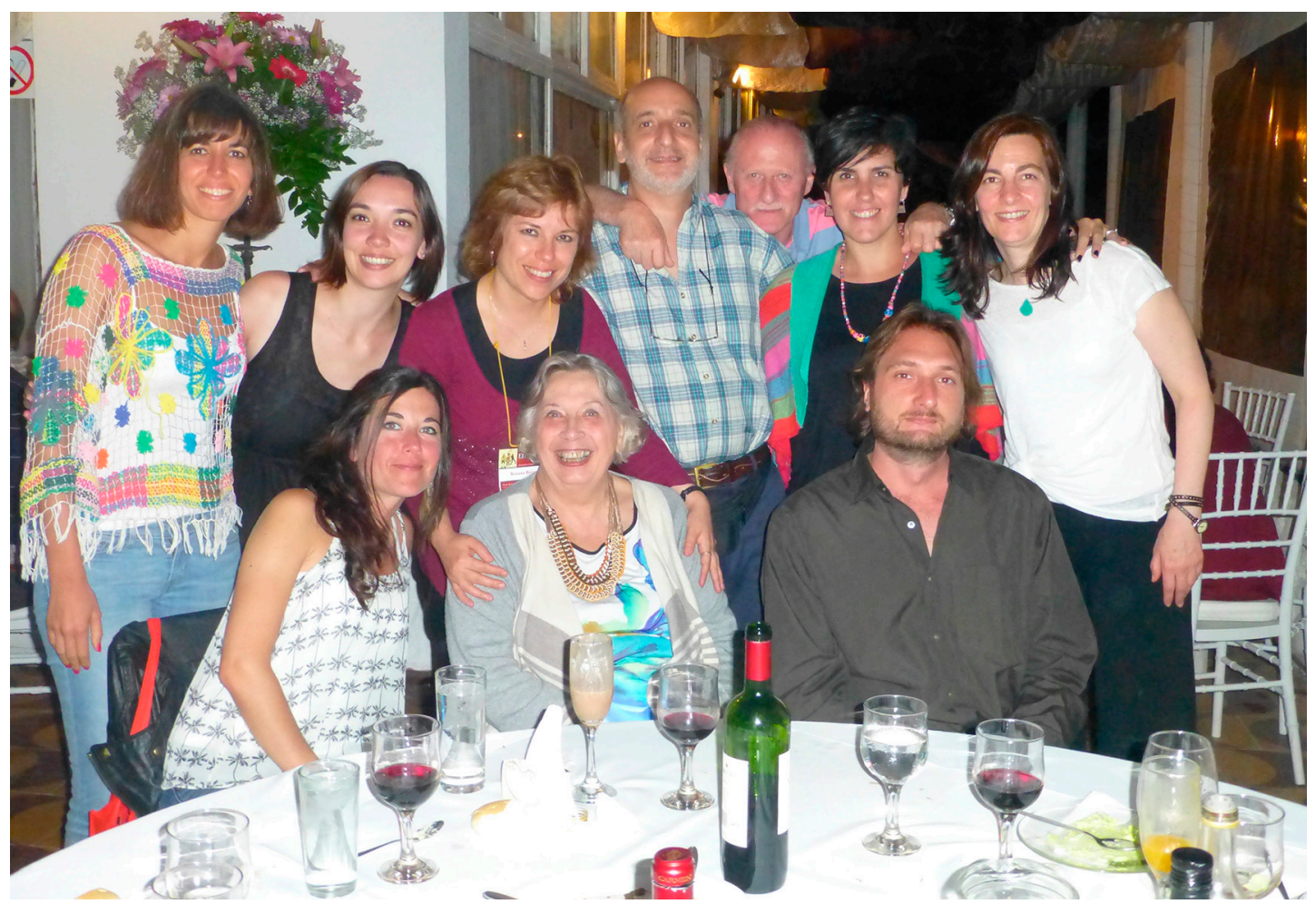

Figura 2. Ana María Lorandi rodeada de algunos de sus discípulos, IX Congreso Internacional de Etnohistoria, Arica. AISE. Gentileza de Lorena Rodríguez.

\section{Referencias Citadas}

Boixadós, R. y C., Bunster.

2016 Prólogo. En Disciplinas sin fronteras. Homenaje a Ana María Lorandi, compilado por R. Boixadós y C. Bunster, pp. 9-14. EFyL, Buenos Aires.

Chiappe, C.

2016 "Imaginarios en pugna. La politización del campo de los estudios andinos en el período fundacional de la etnohistoria andina chilena". Diálogo Andino 49: 283-293.

Chiappe, C.

2018 Entre las luchas pasadas y las presentes. Antecedentes, surgimiento y consolidación de la etnohistoria andina chilena. Tesis doctorado en Antropología. UBA.

Costilla, J.

2016 "Itinerarios antropológicos para una etnohistoria comparada de la religión: cultos católicos americanos en la larga duración". Diálogo Andino 49: 299-309.

Lorandi, A.

2013 “Aprendiendo a investigar". En Los estudios andinos hoy. Práctica intelectual y estrategias de investigación, editado por C. Zanolli et al., pp. 187-202. Prohistoria, Rosario.

Lorandi, A.

2015 Entrevista a A. M. Lorandi realizada en Buenos Aires por A. Ramos y C. Chiappe.

Lorandi, A.

2012 “Etnohistoria, Antropología Histórica o simplemente Historia?”. Memoria Americana 20 (1): 61-78.
Lorandi, A. y M. del Río

1992 Etnohistoria, etnogénesis y transformaciones sociales andinas. Centro Editor de América Latina, Buenos Aires.

Lorandi, A. y R. Rodríguez Molas

1984 "Historia y antropología: hacia una nueva dimensión de la ciencia". Etnia (32): 53-81.

Ramos, A.

2016 El desarrollo de la Etnohistoria andina como campo interdisciplinar: interacciones entre Historia, Arqueología y Antropología, (Perú, Bolivia y Argentina, 1970-2005). Tesis doctoral. Facultad de Filosofía y Letras, Universidad de Buenos Aires, Buenos Aires.

Ramos, A. y C. Chiappe

2015 "Ana María Lorandi y el tren de la etnohistoria". RUNA 37 (2): $97-113$

Taboada, C.

2015 Prólogo. En: Tukuma-Tukuymanta. Los pueblos del búho. Santiago del Estero antes de la Conquista, A, Lorandi, pp. 24-33. Subsecretaría de Cultura de Santiago del Estero, Santiago del Estero.

Torres, S.; M. Gesteira y M. Hirsch

2011 "Entrevista a Ana María Lorandi". Ciclo Encuentros. Publicar IX (XI): 151-165.

Universidad Católica del Norte

1984 "Programa del Simposio de Arqueología Atacameña". San Pedro, 1983. Estudios Atacameños 7. 
Zanolli, C.; A. Ramos; D. Estruch y J. Costilla

2010 Historia, representaciones y prácticas de la etnohistoria en la Universidad de Buenos Aires. Una aproximación antropológica a un campo de confluencia disciplinar. Buenos Aires, Antropofagia.

Zanolli, C.; A. Ramos y C. Chiappe

2016 "Itinerarios académicos de Ana María Lorandi". En Disciplinas sin fronteras. Homenaje a Ana María Lorandi, compilado por R. Boixadós y C. Bunster, pp. 183-217. Buenos Aires, EfyL.

Zanolli, C.; A. Ramos; D. Estruch y J. Costilla

2016 "La etnohistoria en la Universidad de Buenos Aires: un recorrido por 30 años de investigaciones". Diálogo Andino 49: 261-271.

\section{Notas}

1 Presidido por Ana María Lorandi, el Comité contó con Mercedes del Río y Ana María Presta como secretarias.

2 Los nombres que mencionamos provienen del libro de resúmenes y hemos decidido atenernos a ese listado para no ahondar en detalles que se alejan del tema que aquí nos convoca. Sin embargo, es claro que las actas no son un reflejo completo de la realidad, como se desprende de otras fuentes consultadas en el curso de nuestra investigación. Respecto de los asistentes de Chile, sabemos que el trabajo de Hidalgo lo presentó solo la coautora (María

\section{Documentos citados}

Lorandi, A. y M. del Río (30 de diciembre de 1987). [Carta para Gunnar Mendoza]. Archivo Institucional Sección Etnohistoria.

Lorandi, A. y M. del Río (30 de diciembre de 1987). [Carta para John Murra]. Archivo Institucional Sección Etnohistoria.

Murra, J. (22 de enero de 1988) [Carta para Ana María Lorandi] Archivo Institucional Sección Etnohistoria.

1989. Circular No 4 I Congreso Internacional de Etnohistoria. Archivo Institucional Sección Etnohistoria.

1989. Libro de Resúmenes I Congreso Internacional de Etnohistoria. Archivo Institucional Sección Etnohistoria.

Marsilli) y que, aunque los miembros del Grupo Toconce (MAP-UCH) habrían estado presentes encabezados por José Luis Martínez, estos no aparecen en el listado (Chiappe 2018).

3 Al Congreso de 1989 le siguieron los encuentros de Bolivia (1991), Chile (1993), Perú (1996), las dos ediciones argentinas de Jujuy (1998) y Buenos Aires (2005), y los eventos de Perú (2008), Bolivia (2011) y Chile (2014). La sede del próximo congreso será, por primera vez, Ecuador (2018). 
